\title{
La découverte du Trésor de Cluny. Premiers résultats et perspectives de recherche
}

Anne Baud, Anne Flammin et Vincent Borrel

\section{(2) OpenEdition}

10 Journals

Édition électronique

URL : https://journals.openedition.org/cem/15268

DOI : $10.4000 /$ cem. 15268

ISSN : 1954-3093

Éditeur

Centre d'études médiévales Saint-Germain d'Auxerre

Référence électronique

Anne Baud, Anne Flammin et Vincent Borrel, «La découverte du Trésor de Cluny. Premiers résultats et perspectives de recherche ", Bulletin du centre d'études médiévales d'Auxerre | BUCEMA [En ligne], 22.1 | 2018, mis en ligne le 03 septembre 2018, consulté le 22 septembre 2022. URL : http:// journals.openedition.org/cem/15268; DOI : https://doi.org/10.4000/cem.15268

Ce document a été généré automatiquement le 22 septembre 2022.

Creative Commons - Attribution - Pas d'Utilisation Commerciale - Partage dans les Mêmes Conditions 4.0 International - CC BY-NC-SA 4.0

https://creativecommons.org/licenses/by-nc-sa/4.0/ 


\title{
La découverte du Trésor de Cluny. Premiers résultats et perspectives de recherche
}

\author{
Anne Baud, Anne Flammin et Vincent Borrel
}

La nouvelle campagne de fouille, qui s'est déroulée dans l'abbaye de Cluny durant l'automne 2017, a donné lieu à la découverte exceptionnelle d'un trésor monétaire, comprenant également une bague sigillaire et un petit carré d'or. Il s'agissait de la troisième opération sur le site de l'ancienne infirmerie. Celle-ci,

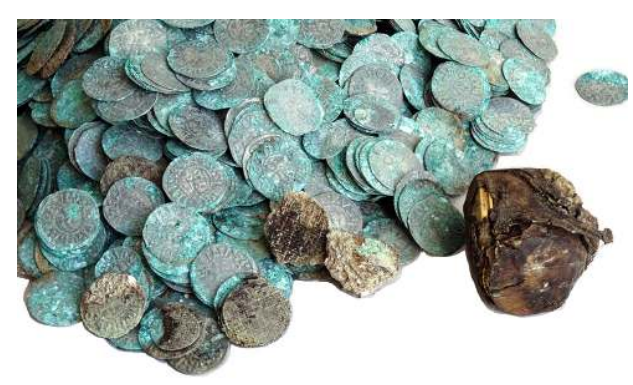
complètement détruite à l'occasion des grands travaux de reconstruction au milieu du xvIII ${ }^{\mathrm{e}}$ siècle, était essentiellement connue par le plan anonyme daté de 1700 (fig. 1). 
Fig. 1 - Cluny (71), abbaye, plan anonyme daté vers 1700 avec la localisation de l'infirmerie du trésor

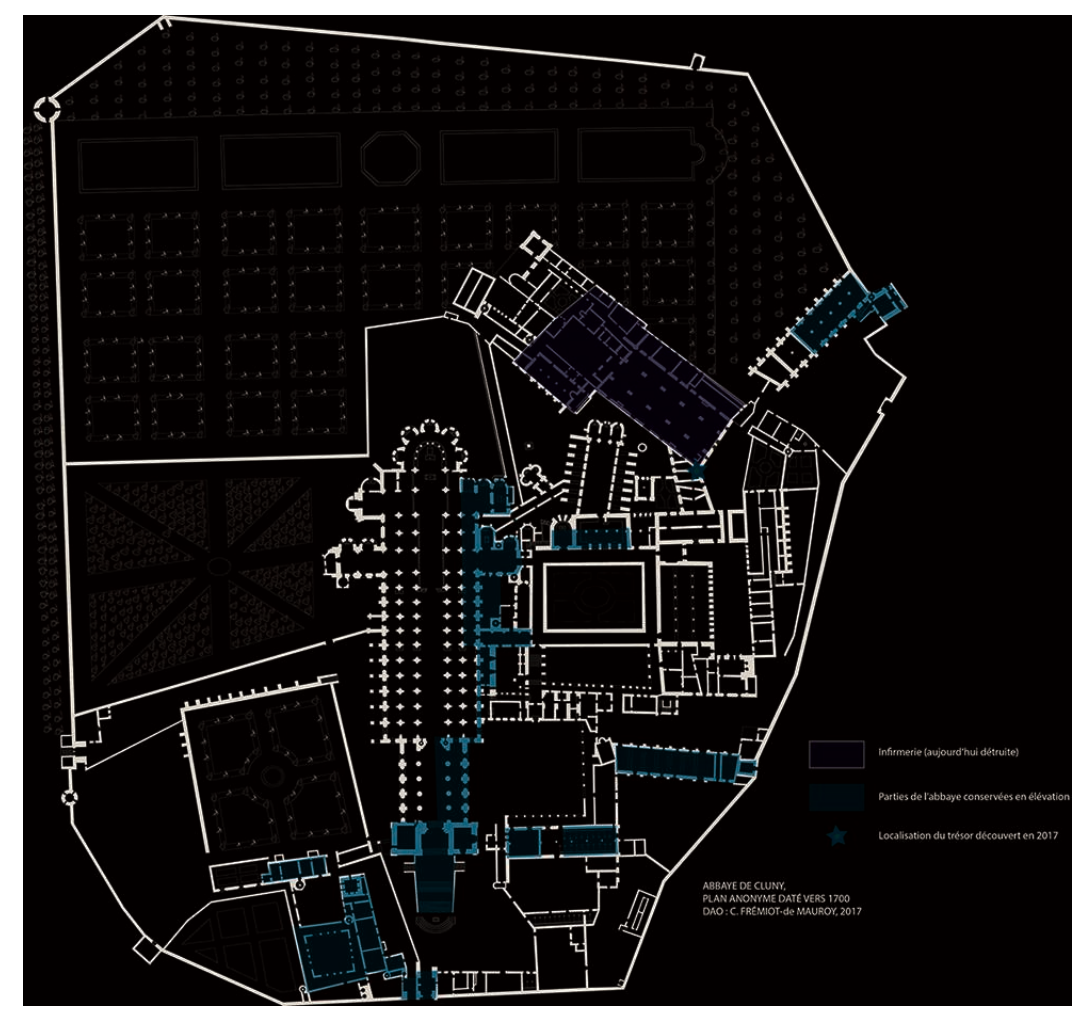

DAO C. Frémiot-de Mauroy

2 Aucune recherche archéologique n'avait été entreprise jusqu'alors à cet endroit. L'archéologue américain K. J. Conant s'est davantage intéressé à l'histoire des églises, et les restitutions de l'infirmerie monastique, dans son ouvrage en 1968, reposent uniquement sur une interprétation des sources textuelles et iconographiques ${ }^{1}$. Les opérations archéologiques menées entre 2011 et 2013 (en cours de publication), en collaboration avec Christian Sapin et le Centre d'études médiévales d'Auxerre, ont mis au jour la chapelle dédiée à la Vierge. Cette église de la fin du $x^{\mathrm{e}}$ siècle, destinée aux malades, avait été reconstruite à la fin $\mathrm{du} \mathrm{XI}^{\mathrm{e}}$ siècle. Plus grande, elle était mieux adaptée à la communauté monastique qui ne cessait de s'accroître. D'après le plan anonyme et les textes, la chapelle se trouvait à proximité immédiate de l'infirmerie. Les résultats de ces fouilles ont ainsi suscité en 2015 un nouveau programme de recherche, qui s'articule autour de la place des malades à Cluny.

3 Le plan anonyme localise, au sud-est du chevet de l'église, une vaste infirmerie composée de différents bâtiments et de cours qu'il est nécessaire de mieux comprendre. Les fouilles, menées avec les étudiants de l'université Lyon $2^{2}$, ont concerné les espaces situés au nord-est de la grande salle, dite «salle de Pierre le Vénérable ». Elles ont permis de mettre au jour plusieurs états du XI ${ }^{\mathrm{e}}$ au XVIII ${ }^{\mathrm{e}}$ siècle. La «salle de Pierre le Vénérable » ne pouvant être fouillée en raison de sa position sous les voies actuelles et de la présence d'égouts modernes, un sondage de $8 \mathrm{~m}$ sur $6 \mathrm{~m}$ a été ouvert à la pelle mécanique afin de vérifier la position de son angle sud-ouest. À environ 0,80 m sous le niveau du sol des jardins actuels et à l'attitude de 237,21 m NGF (niveau supérieur), le godet de la pelle a effleuré le dépôt monétaire entraînant un arrêt instantané du décapage. Pour différents facteurs liés à sa position très instable 
dans la berme ou à l'heure tardive de la découverte, il n'a pas été possible de prélever la motte dans laquelle il se trouvait en vue d'une fouille en laboratoire. À défaut, le démontage du trésor fut documenté par une couverture photographique (fig. 2).

Fig. 2 - Vue du trésor en cours de fouille

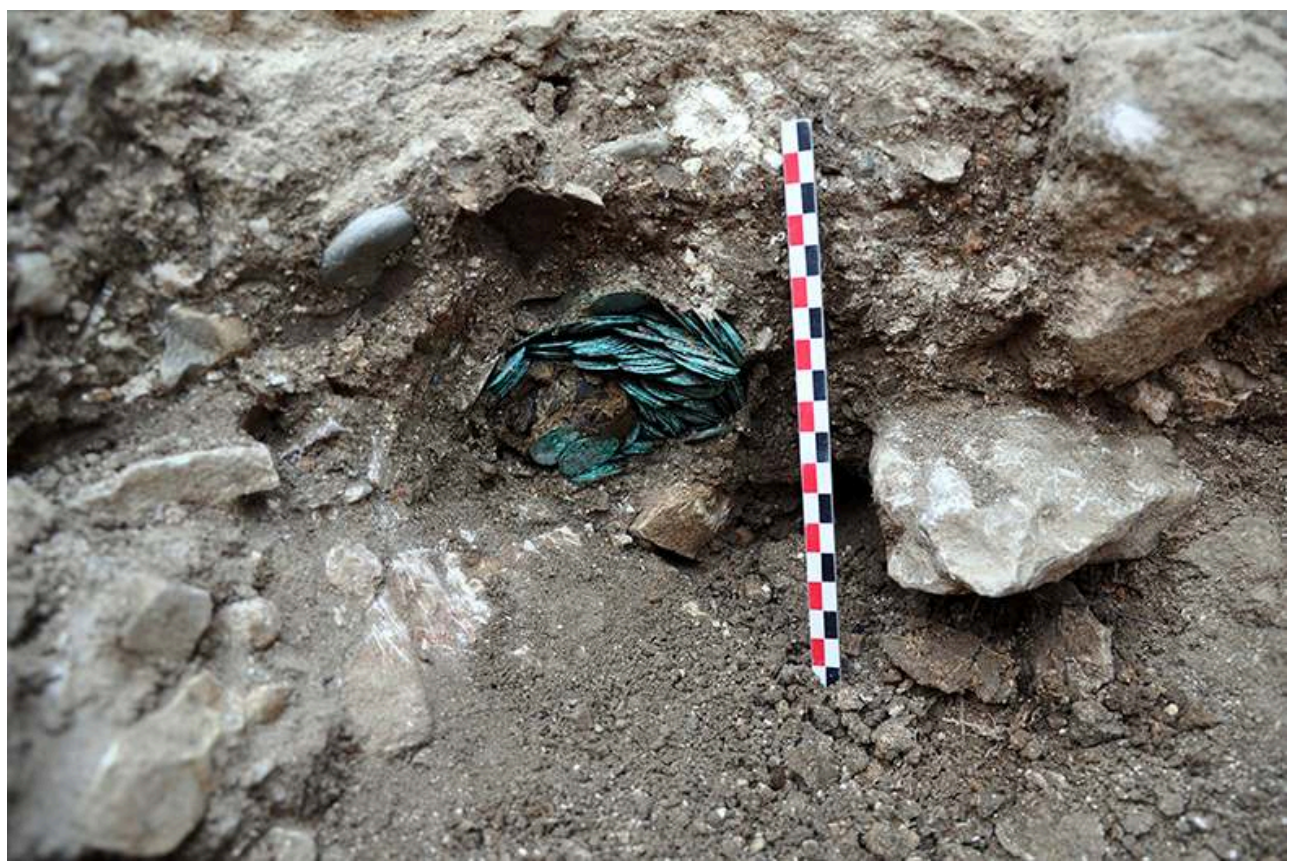

Cl. A. Baud

Par la suite, un petit sondage a été ouvert au-dessus du trésor pour renseigner les conditions du dépôt. Celui-ci se trouvait sous un sol extrêmement lacunaire, dans un bâtiment reliant l'infirmerie aux bâtiments conventuels.

\section{La composition du trésor}

Le trésor, déposé au Centre de restauration et d'études archéologiques municipal de Vienne (38) pour nettoyage et consolidation, était composé de 2113 deniers clunisiens et de 143 oboles enveloppés dans un tissu dont les trames ont été minéralisées sur les faces externes des monnaies placées à la périphérie du contenant ${ }^{3}$. Au centre, une petite bourse en peau contenait une bague sigillaire accompagnée de 21 dinars arabes en or, un « carré d'or » composé de feuilles d'or repliées, placé dans une petite poche en cuir et une piécette dans le même métal précieux (fig. 3). 
Fig. 3 - Bourse en peau avant son ouverture

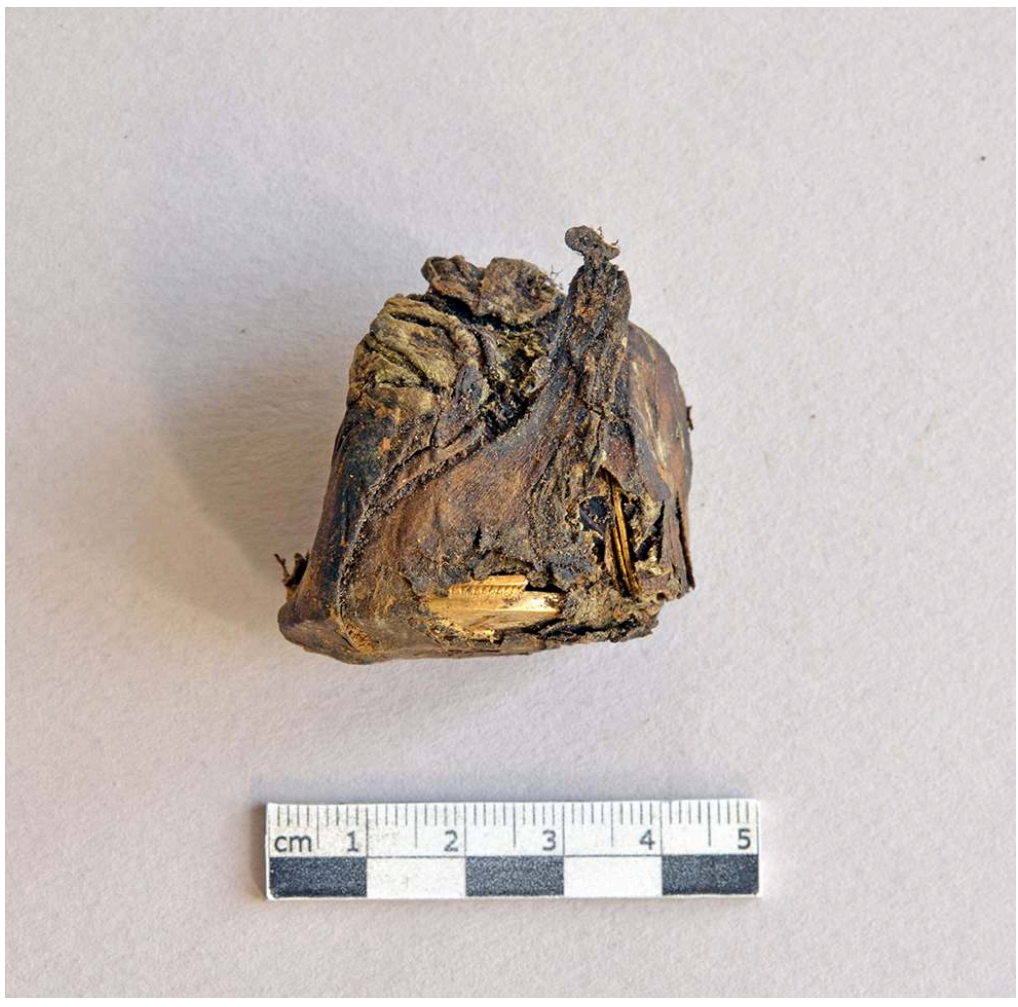

Cl. CREAM

6 Les monnaies et le " carré d'or » étaient très soigneusement emboîtés contre l'anneau de la bague sigillaire. Les premières analyses, effectuées rapidement par différents spécialistes lors de la trouvaille, permettent aujourd'hui une première vision d'ensemble (fig. 4). 
Fig. 4 - Carte représentant l'origine des monnaies

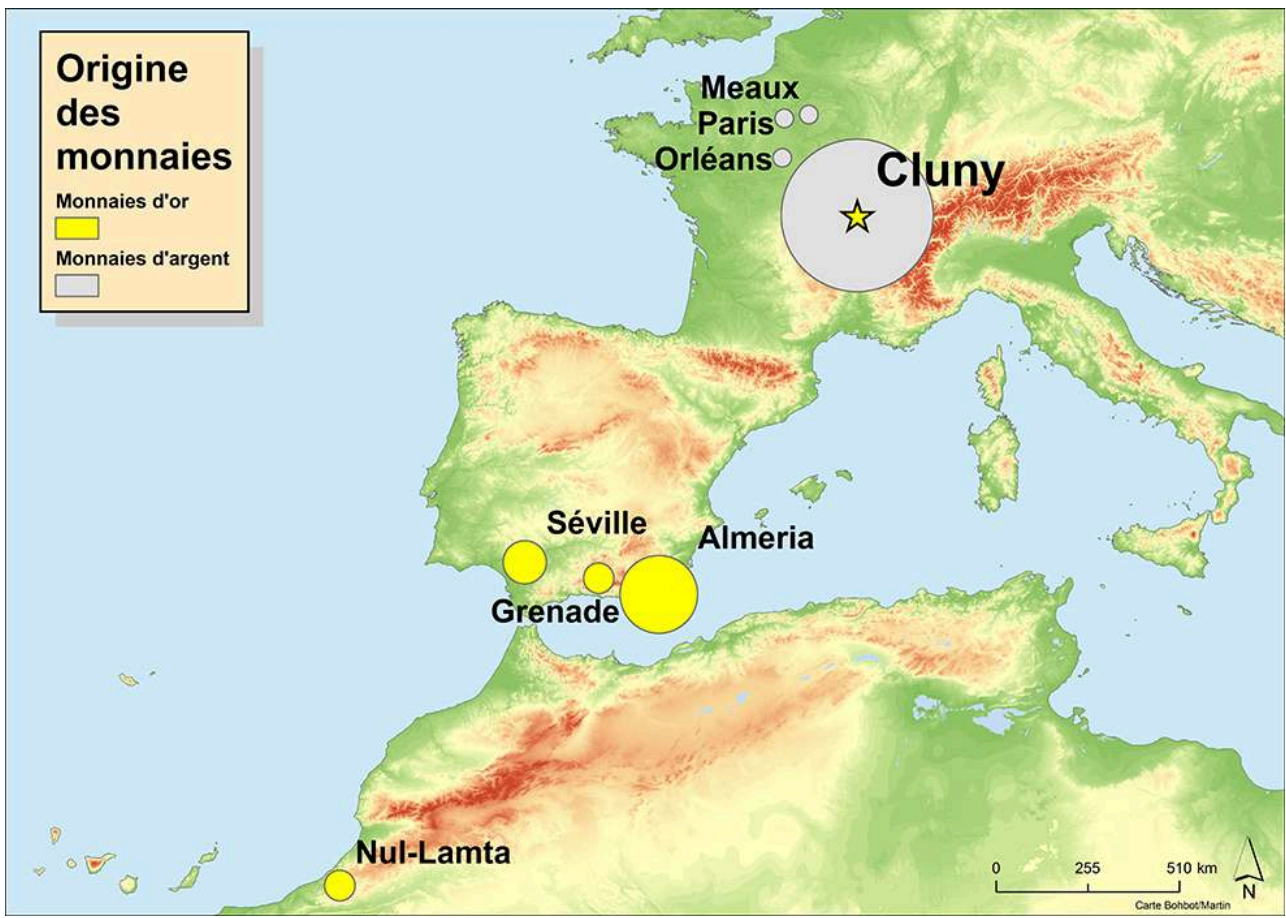

Conception et DAO V. Borrel

\section{Les monnaies d'argent}

C'est la première fois qu'est mis au jour un nombre aussi important de monnaies clunisiennes (fig. 5).

Fig. 5 - Trésor, deniers clunisiens en argent et la bourse en peau

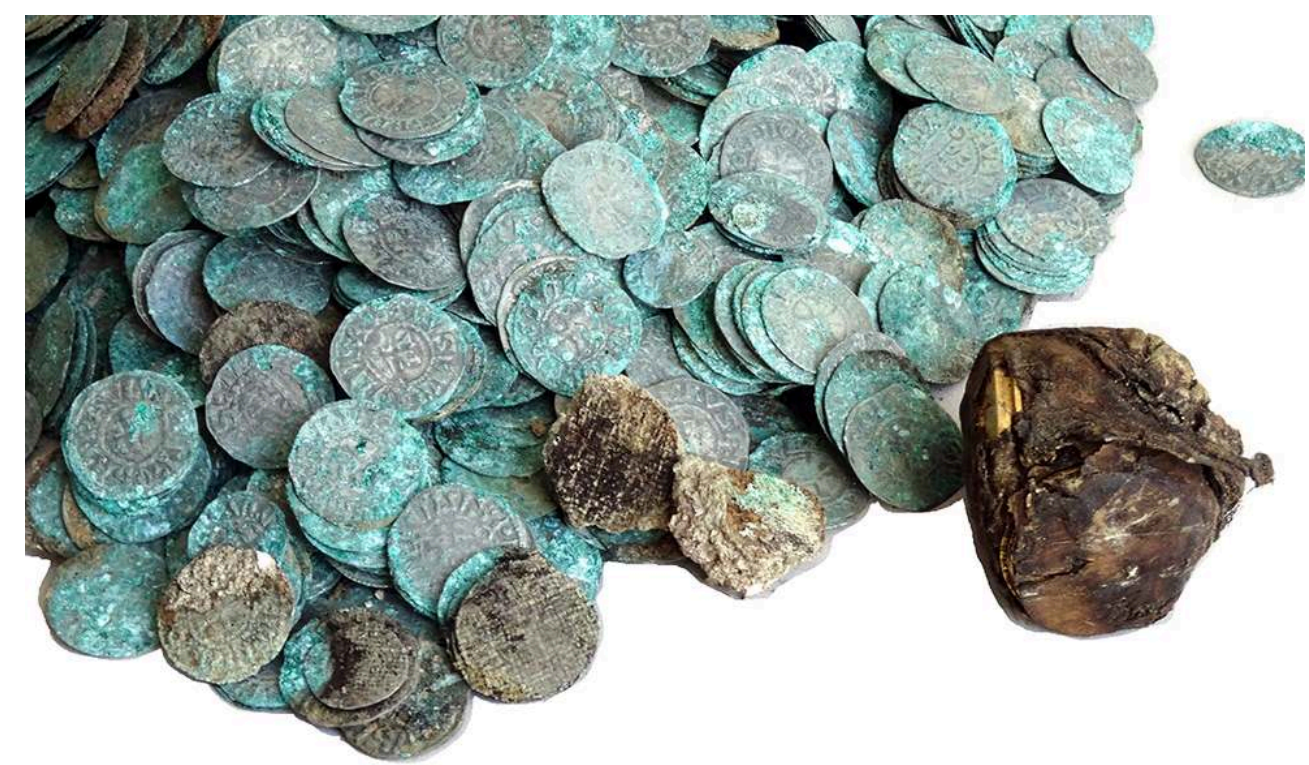

Cl. V. Borrel 
L'ensemble des pièces est assez bien conservé. Quelques concrétions ont parfois aggloméré en piles les deniers et les oboles, mais les monnaies restent facilement identifiables. Au droit, figurent les noms des saints Pierre et Paul, et la clé, attribut du premier. Au revers, la mention CLVNIACO CENOBIo désigne l'émetteur. Le type même du denier clunisien n'évoluera que très peu durant toute la période d'émission, qui s'étend jusqu'au XIII siècle. Les séries sont principalement repérables aux nombres de "dents " de la clé. Les plus anciennes, émises dès la fin du XI $\mathrm{x}^{\mathrm{e}}$ jusqu'au milieu du XII ${ }^{\mathrm{e}}$ siècle, et auxquelles appartiennent les monnaies du trésor, en comportent cinq. Trois deniers, émis dans le royaume de France, font figure d'exception dans la masse de monnaies clunisiennes. Le premier a été frappé à Meaux par l'évêque Burchard (1120-1134). Les deux autres ont été émis à Orléans et à Paris par le roi de France et sont actuellement attribués à Louis VII (1137-1180). Ces monnaies, appartenant aux systèmes monétaires parisis et champenois, étaient susceptibles de circuler en Bourgogne à équivalence avec les monnaies locales.

\section{Les dinars almoravides ou marabotins}

Les dinars ont tous été émis par les Almoravides (fig. 6), dynastie régnant à la fois sur Al-Andalus et le Maghreb. Vingt dinars sont au nom de l'émir Ali ben Youssef (500-537 $\mathrm{AH} / 1106-1143)$ et, le plus récent, daté de $539 \mathrm{AH}$ (1144), porte le nom de son fils, Tashfin, qui n'a régné que deux ans (537-539 AH/1143-1145).

Fig. 6 - Trésor, monnaie en or de l'atelier d'Almeria en Espagne

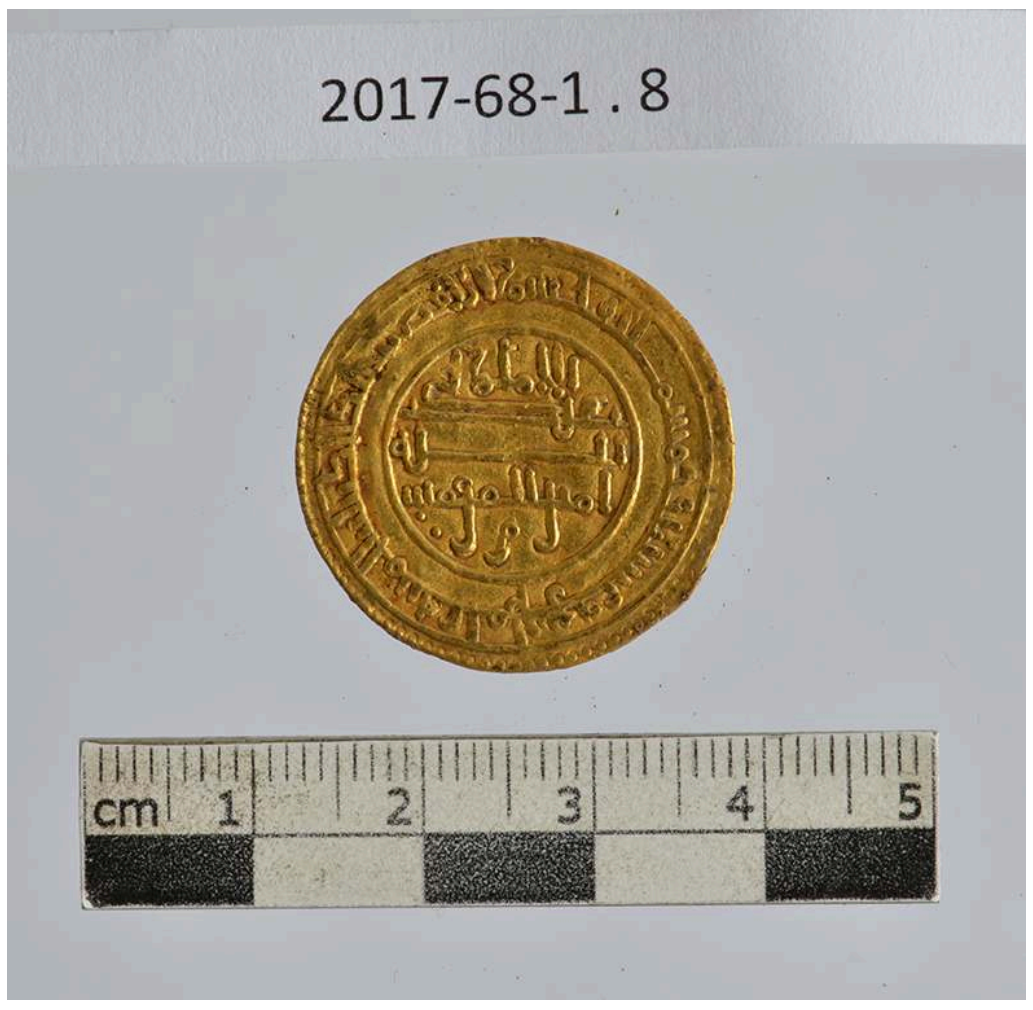

Cl. CREAM

10 Quatre ateliers ont été identifiés : trois dans la péninsule ibérique (Almeria, Grenade et Séville) et le dernier, nettement plus méridional, dans une cité aujourd'hui disparue au 
sud du Maroc: Nul-Lamta ${ }^{4}$. Au XII siècle, dans l'état actuel de nos connaissances, hormis dans les zones en contact direct avec les Musulmans, à savoir le royaume de Sicile et l'Espagne chrétienne, il n'y a pas d'émission de monnaies d'or en Europe. L'or n'est véritablement de nouveau produit en masse hors de ces deux zones que lorsque Florence commence à émettre le florin, dès 1252 . De ce fait, il n'existe a priori pas, au XII ${ }^{e}$ siècle, de monnaies de forte valeur nécessaires aux transactions importantes et qui pourraient permettre de s'affranchir du transport de masses toujours plus importantes de deniers ${ }^{5}$. Depuis la fin du $\mathrm{x}^{\mathrm{e}}$ siècle, en effet, face à la demande croissante de monnaies, la tendance générale est à l'affaiblissement de la teneur en argent des deniers. Le métal est généralement allié à des quantités de plus en plus conséquentes de cuivre, ce qui entraîne, à masse d'argent constante, l'augmentation de la masse monétaire finale. Le problème est pallié plus tard par l'utilisation de lingots d'argent pur, et, dans une certaine mesure, par l'utilisation d'or.

11 Ces dinars apparaissent dans la documentation chrétienne sous le nom de marabotins, par déformation d'al-morabitin, du nom des Almoravides, qui sont en réalité les principaux pourvoyeurs des monnaies d'or circulant au nord des Pyrénées. C'est d'ailleurs sous ce nom que, le 29 juillet 1142, «l'empereur des Espagnes » Alphonse désigne ces monnaies dans le diplôme qu'il concède à Pierre le Vénérable, alors en voyage dans la péninsule ${ }^{6}$.

\section{Le « carré d'or »}

12 Ce que nous avons appelé, dans un premier temps et par commodité, le "carré d'or ", mesure $28 \mathrm{~mm}$ de côté, $5 \mathrm{~mm}$ d'épaisseur maximum et pèse $24 \mathrm{gr}$ (fig. 7).

Fig. 7 - Trésor, lingot d'or

2017-68-1. 26
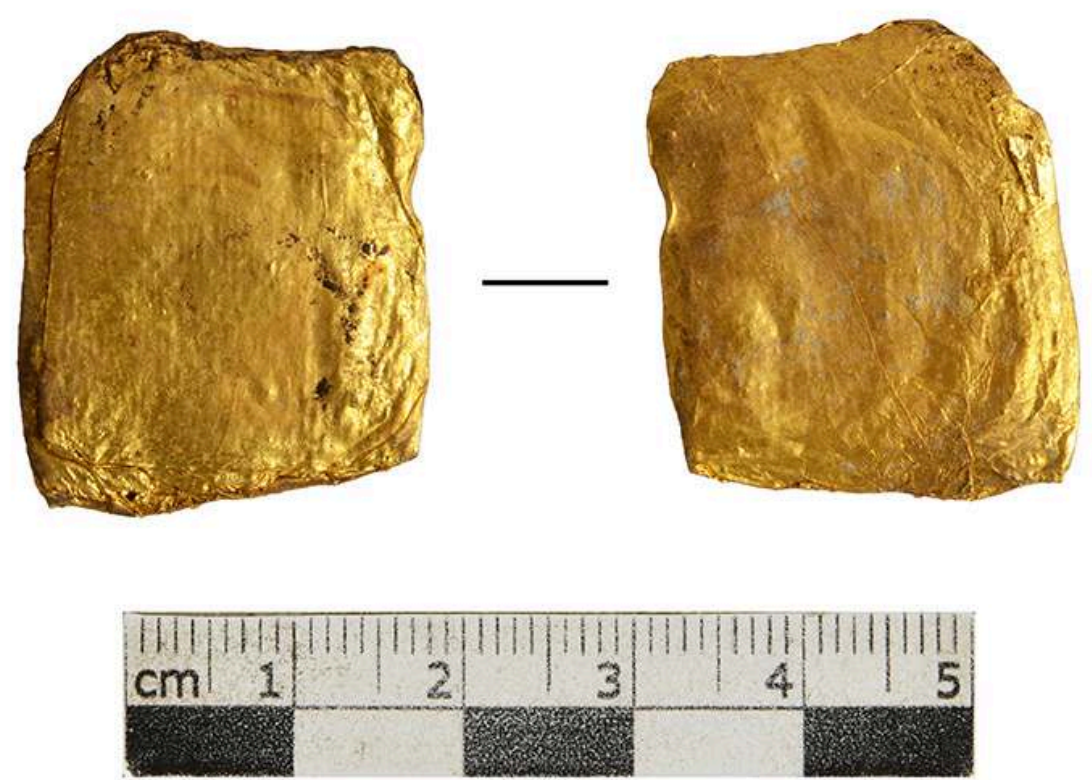
Composé de feuilles d'or repliées sur elles-mêmes, il était impossible de déterminer si celles-ci enveloppaient un objet ou bien s'il s'agissait tout simplement d'un lingot d'or. Le carré d'or fut ainsi confié par l'intermédiaire de Christian Vernou au laboratoire d'ARC-Nucléart à Grenoble? ${ }^{7}$. La densité de l'or constituant une réelle difficulté, ce laboratoire fut en mesure d'utiliser, grâce à une opportunité exceptionnelle, les capacités du Synchrotron à l'ESRF (European Synchrotron Radiation Facility) situé à proximité du CEA de Grenoble ${ }^{8}$. L'objet fut soumis à une analyse à base de microtomographie à rayon X (250 kiloélectrons-volts et 268 kiloélectrons-volts). Cette analyse permit de confirmer que le " carré d'or » était formé d'une ou plusieurs feuilles pliées sur elles-mêmes, sans objet interne. Il s'agit ainsi d'un petit lingot d'or associé à des monnaies et un anneau sigillaire.

\section{L'anneau sigillaire}

14 La plus belle pièce du trésor est sans aucun doute l'anneau sigillaire en or (27 gr), comprenant un large chaton au centre duquel se trouve une intaille antique en cornaline (fig. 8).

Fig. 8 - Trésor, bague sigillaire
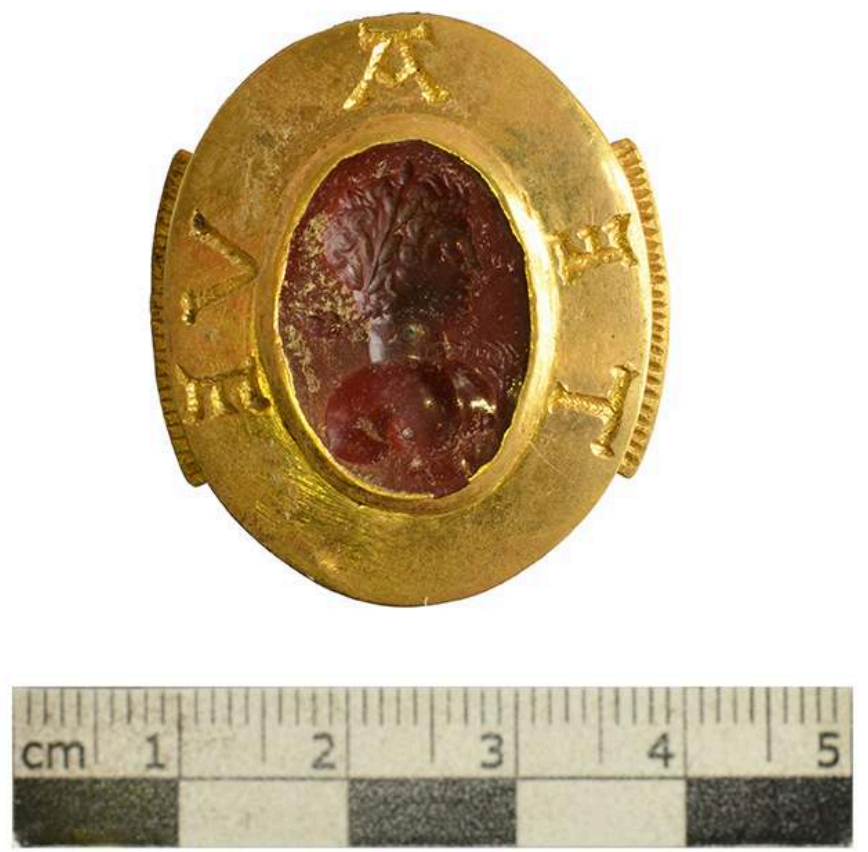

CI. CREAM

Celle-ci figure, selon François Planet, l'empereur Caracalla sous les traits du demi-dieu Hercule 9 . Autour de l'intaille est gravée, dans le cerclage en or, l'inscription /A VE TE /. Les anneaux sigillaires sont couramment attestés au Moyen Âge entre le $\mathrm{xI}^{\mathrm{e}}$ et le $\mathrm{xIII}^{\mathrm{e}}$ siècle $^{10}$. Bien que l'étude ne soit qu'à peine commencée, nous savons que 
l'importance de l'anneau et l'inscription désignent comme détenteur du bijou un haut dignitaire ecclésiastique ou religieux.

\section{Une piécette d'or}

16 Cette petite pastille d'or ( $1,22 \mathrm{~g})$ striée est finalement l'élément le plus mystérieux de cet ensemble, car, à l'heure actuelle, aucune hypothèse n'a encore pu être formulée quant à son rôle et à la raison de sa présence dans le trésor (fig. 9).

Fig. 9 - Trésor, piécette d'or

\section{7-68-1 . 24}
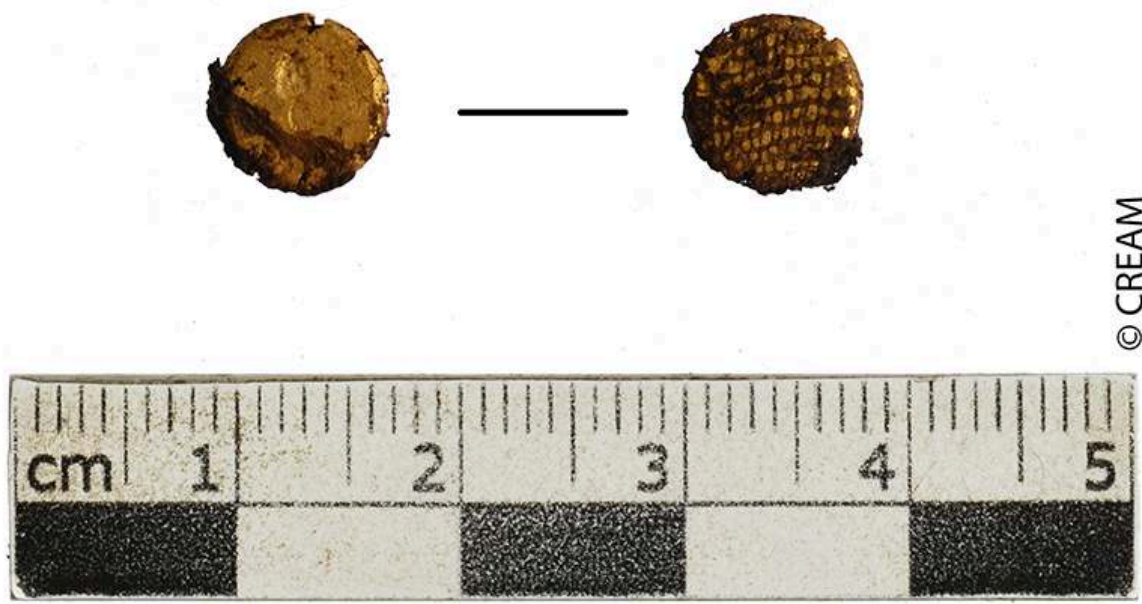

\section{Cl. CREAM}

\section{Premiers éléments de réflexions}

Si l'importance du trésor de Cluny n'est pas à démontrer, il faut néanmoins en souligner les raisons. En premier lieu, c'est la première fois que l'on retrouve un trésor du XII ${ }^{e}$ siècle dans une abbaye. Découvert sous un sol d'un bâtiment aujourd'hui détruit, il fut enterré et jamais retrouvé. Trésor oublié, alliant à la fois objets et monnaies d'or et d'argent, il pose de nombreuses questions sur les motifs de son enfouissement et sur l'identité de son propriétaire. Le trésor est complet et l'assemblage des différents éléments, dans divers contenants, offre des pistes importantes pour son interprétation.

L'examen des autres trésors contenant des dinars montre que le trésor de Cluny forme un ensemble particulièrement cohérent. En effet, le trésor d'Aurillac, avec 49 monnaies, incluait des dinars almoravides, mais également des exemplaires des souverains de 
Murcie, un dinar almohade et quatre dinars hubides ${ }^{11}$. Celui d'Albon comportait des monnaies de Marrakech, de Grenade, mais également d'Alexandrie ${ }^{12}$.

Les marabotins de Cluny ont été émis sous l'autorité des seuls Almoravides sur une période de vingt-quatre ans, entre 1120 (514 AH) et 1144 (539 AH). Compte tenu des liens privilégiés de l'abbaye avec l'Espagne, les monnaies arabes pourraient directement être parvenues en Bourgogne depuis la péninsule Ibérique, sans passer par le circuit commercial régional. Concernant cette fois-ci les deniers clunisiens, peu d'entre eux ont été retrouvés isolément, alors qu'ils ont circulé dans une grande partie de l'Europe et même jusque vers Northampton en Angleterre. Fait surprenant, hormis le trésor de 2017, les plus importantes trouvailles ont été réalisées non pas en Bourgogne mais en Espagne, respectivement à Sahagún (150 deniers) et Astorga (environ 7 deniers) ${ }^{13}$. Si les deux sites étaient des étapes stratégiques sur le chemin de Saint-Jacques-de-Compostelle, Sahagún était également le lieu d'implantation d'un prieuré de Cluny et un lieu d'émigration de colons venus de France, d'Allemagne et de Bourgogne.

Le bâtiment dans lequel a été caché ce trésor est encore mal connu. Reliant l'infirmerie au couvent, il referme un espace situé entre la chapelle mariale et la grande salle de l'infirmerie. Ce lieu, à l'intérieur de la clôture, n'était accessible qu'aux religieux ou à leurs familiers. Le détenteur du trésor était sans doute malade lorsqu'il a caché son bien, peut-être se rendait-il à l'infirmerie. Il est vraisemblablement mort avant qu'il ne puisse le récupérer. Ou bien ce trésor fut-il dérobé au sein du monastère, caché et jamais récupéré.

21 L'étude du trésor est actuellement en cours. Les monnaies vont faire l'objet d'analyses approfondies, afin d'obtenir de plus amples informations sur le monnayage clunisien et sur la circulation des pièces d'or en Occident au xII ${ }^{e}$ siècle, notamment entre l'Espagne et Cluny. L'examen de la peau de la petite bourse devrait permettre d'identifier l'espèce animale. Les composantes de l'objet le plus précieux du trésor, l'anneau sigillaire, seront soigneusement analysées: l'intaille antique (matériau et iconographie), l'or (origine), l'inscription (étude épigraphique...). L'objectif de la fouille de l'automne 2018 sera de documenter l'endroit précis où le trésor a été déposé afin de mieux saisir sa fonction sous l'abbatiat de Pierre le Vénérable.

Reçu : 14 juin 2018 - Accepté : 20 juin 2018

\section{NOTES}

1. K.-J. Conant, Cluny, la maison du chef d'Ordre, Mâcon, 1968.

2. Les fouilles archéologiques s'appuient aussi sur l'UMR 5138 « Archéologie et archéométrie ».

3. Le coût de la restauration a été pris en charge par le Service régional de l'archéologie Bourgogne Franche-Comté.

4. Cette première étude a été réalisée par Marc Parverie, numismate travaillant sur les monnayages musulmans ayant circulé en France. Nos remerciements vont à Messieurs Tawfiq Ibrahim et Mohamed El Hadri pour la confirmation des datations. 
5. Regesta comitum sabaudiae, $\mathrm{n}^{\circ} 194$ : En 1147, le monastère Saint-Just de Suse donne 11000 sous au comte de Savoie Amédée III sur le point de partir à la croisade. En comptant 1,1 g par denier, cette somme représente une masse d'environ $145 \mathrm{~kg}$.

6. Chartes de Cluny, t. $5, n^{\circ} 4072$ : Dono etiam eidem acclesice et abbati prenominato ducentorum morabotinorum redditum in balneis que in Burgis sunt.

7. Nous remercions très chaleureusement Christian Vernou et Paul Tafforeau pour avoir effectué cette analyse.

8. Le CEA de Grenoble est un des centres de recherche du Commissariat à l'énergie atomique.

9. François Planet est conservateur au musée des Beaux-Arts de Lyon et chargé du médaillier.

10. Jean-Luc Chassel (maître de conférences à l'université de Paris-Nanterre) a procédé à une première analyse de la bague.

11. F. CLÉMENT, «Les monnaies arabes et à légende arabe trouvées dans le Grand Ouest ", Annales de Bretagne et des Pays de l'Ouest, 115/2 (2008), p. 159-187.

12. J. DuPLESSY, Les trésors monétaires médiévaux et modernes découverts en France, t. 1 (751-1223), Paris, 1985, n 15. L'emplacement du trésor est donné à Anneyron, mais le lieu exact a été rectifié par Jean-Michel Poisson : il s'agit du lieu-dit les Marrons, qui ne se trouve pas sur la commune d'Anneyron, mais sur celle d'Albon.

13. Concernant Sahagún: M. DHÉNIN et R. PROT, «Deniers normands dans un trésor espagnol (xII ${ }^{\mathrm{e}}$ siècle) ", Bulletin de la Société française de numismatique, 51/4 (1996), p. 75-79. Ce trésor, contenant probablement plusieurs milliers de monnaies, a été dispersé entre la France et l'Espagne et il faut, pour le moment, se contenter de cette seule publication succincte. Concernant Astorga : J. Colino, Una monedas del Camino de Santiago, León, s. d.

INDEX

Mots-clés : trésor, bague sigillaire, monnaies médiévales, Moyen Âge, or

\section{AUTEURS}

\section{ANNE BAUD}

Maître de conférences, université Lyon 2, UMR 5138

ANNE FLAMMIN

Ingénieur d'étude CNRS, UMR 5138

VINCENT BORREL

Doctorant ENS, UMR 8546 AorOc 